\title{
SPAR - a randomised, placebo-controlled phase II trial of simvastatin in addition to standard chemotherapy and radiation in preoperative treatment for rectal cancer: an AGITG clinical trial
}

Michael B. Jameson ${ }^{1}$, Kirsten Gormly², David Espinoza ${ }^{3}$, Wendy Hague ${ }^{3}$, Gholamreza Asghari ${ }^{4}$, Grahame Mark Jeffery ${ }^{5}$, Timothy Jay Price ${ }^{6}$, Christos Stelios Karapetis ${ }^{7}$, Michael Arendse ${ }^{8}$, James Armstrong ${ }^{9}$, John Childs ${ }^{10}$, Frank A. Frizelle ${ }^{11}$, Sam Ngan ${ }^{12}$, Andrew Stevenson ${ }^{13}$, Martinus Oostendorp ${ }^{3}$ and Stephen P. Ackland ${ }^{14^{*}}$ (D)

\begin{abstract}
Background: Retrospective studies show improved outcomes in colorectal cancer patients if taking statins, including overall survival, pathological response of rectal cancer to preoperative chemoradiotherapy ( $\mathrm{pCRT}$ ), and reduced acute and late toxicities of pelvic radiation. Major tumour regression following pCRT has strong prognostic significance and can be assessed in vivo using MRI-based tumour regression grading (mrTRG) or after surgery using pathological TRG (pathTRG).

Methods: A double-blind phase 2 trial will randomise 222 patients planned to receive long-course fluoropyrimidine-based PCRT for rectal adenocarcinoma at $18+$ sites in New Zealand and Australia. Patients will receive simvastatin $40 \mathrm{mg}$ or placebo daily for 90 days starting 1 week prior to standard pCRT. Pelvic MRI 6 weeks after pCRT will assess mrTRG grading prior to surgery. The primary objective is rates of favourable (grades 1-2) mrTRG following pCRT with simvastatin compared to placebo, considering mrTRG in 4 ordered categories $(1,2,3,4-5)$. Secondary objectives include comparison of: rates of favourable pathTRG in resected tumours; incidence of toxicity; compliance with intended pCRT and trial medication; proportion of patients undergoing surgical resection; cancer outcomes and pathological scores for radiation colitis. Tertiary objectives include: association between mrTRG and pathTRG grouping; inter-observer agreement on mrTRG scoring and pathTRG scoring; studies of T-cell infiltrates in diagnostic biopsies and irradiated resected normal and malignant tissue; and the effect of simvastatin on markers of systemic inflammation (modified Glasgow prognostic score and the neutrophillymphocyte ratio). Trial recruitment commenced April 2018.

Discussion: When completed this study will be able to observe meaningful differences in measurable tumour outcome parameters and/or toxicity from simvastatin. A positive result will require a larger $\mathrm{RCT}$ to confirm and validate the merit of statins in the preoperative management of rectal cancer. Such a finding could also lead to studies of statins in conjunction with chemoradiation in a range of other malignancies, as well as further exploration of possible mechanisms of action and interaction of statins with both radiation and chemotherapy. The translational substudies undertaken with this trial will (Continued on next page)
\end{abstract}

\footnotetext{
* Correspondence: stephen.ackland@newcastle.edu.au

${ }^{14}$ University of Newcastle, Lake Macquarie Private Hospital and Calvary Mater

Newcastle Hospital, Newcastle, Australia

Full list of author information is available at the end of the article
}

(c) The Author(s). 2019 Open Access This article is distributed under the terms of the Creative Commons Attribution 4.0 International License (http://creativecommons.org/licenses/by/4.0/), which permits unrestricted use, distribution, and reproduction in any medium, provided you give appropriate credit to the original author(s) and the source, provide a link to the Creative Commons license, and indicate if changes were made. The Creative Commons Public Domain Dedication waiver (http://creativecommons.org/publicdomain/zero/1.0/) applies to the data made available in this article, unless otherwise stated. 


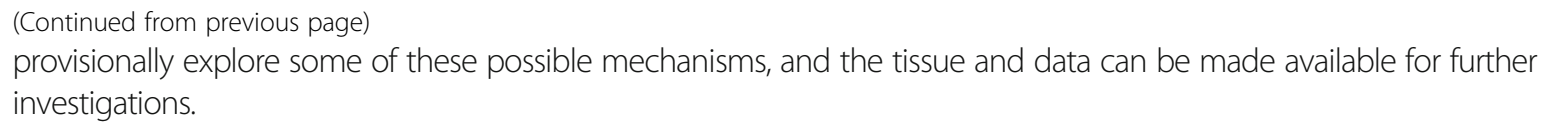

Trial registration: ANZ Clinical Trials Register ACTRN12617001087347.

(www.anzctr.org.au, registered 26/7/2017)

Protocol Version: 1.1 (June 2017).

Keywords: Rectal cancer, Chemoradiation, Statins, HMG-coA reductase inhibitor, Tumour regression grading

\section{Background}

Summary of clinical condition and current treatments

Colorectal cancer (CRC) is common: 3016 cases were diagnosed in New Zealand (NZ) in 2012 with 1283 deaths [1] and rectal cancer represents about one-third of all colorectal cancers in NZ [2]. In Australia, 5114 rectal cancer cases were diagnosed in 2011 with 2018 deaths in 2012 [3]. Rectal cancer usually presents with locally-advanced T3 disease that requires 'short course' radiotherapy (SCRT) or, more commonly, 'long course' preoperative chemoradiation (pCRT - in which either infusional 5-fluorouracil (5FU) or oral capecitabine are administered concurrently with radiotherapy) for 5-6 weeks before surgery, and often adjuvant post-operative chemotherapy. While these advances in the management of resectable rectal cancer have reduced local relapse to $<10 \%$ in most patients, those with higher tumour stage, or evidence on staging MRI scan of invasion of local nodes, mesorectal fascia or blood vessels, have substantially higher local relapse rates and poorer overall survival (OS) [4]. In addition, distant relapse still occurs in $25-30 \%$ of patients, with most dying within 5 years [5]. Adding more drugs (such as oxaliplatin or irinotecan) to pCRT increases toxicities but with no improvement in cancer outcomes [6]. Other strategies are being explored in phase 2 and 3 trials but none have yet changed the standard of pCRT (or, less commonly, SCRT).

Unfortunately, the majority of patients (about 60\%) with high-risk tumours have poor responses of their tumour to pCRT, and this group have double the risk of relapse compared to good responders [7]. Furthermore about $10 \%$ of surviving patients suffer from long-term significant bowel toxicity from RT. [5, 8] There is a clear need for improved efficacy and reduced toxicity in the large number of rectal cancer patients treated with pCRT every year in NZ and Australia.

\section{Summary of findings from pertinent pre-clinical studies and clinical trials}

Statins offer the opportunity to improve outcomes in the treatment of rectal cancer. A Danish population study of 295,925 cancer cases of all types revealed that the use of statins significantly improved overall survival (OS) and specifically in those with CRC (HR 0.79, 95\% CI 0.740.85) [9]. Similarly, a registry study of 10,762 CRC patients from Taiwan reported that, on multivariate analysis, cancer-specific survival was independently and significantly improved in statin users (HR $0.72 p<0.001$ ) [10]. More recently a population-based cohort study of 7657 patients with CRC in the United Kingdom showed that statin use improved cancer-specific survival (HR, $0.71 ; 95 \% \mathrm{CI}, 0.61-0.84$ ) and all-cause mortality (HR, 0.75; 95\% CI, 0.66-0.84) [11].

Preclinical studies have elaborated effects on cell signalling pathways that may contribute to better cancer outcomes with statins, many of which are independent of cholesterol metabolism [12]:

- generation of pro-apoptotic, growth-inhibitory and pro-differentiation responses in tumours;

- inhibition of angiogenesis, invasion and metastasis;

- reducing inflammation and inhibiting radiation (RT)-induced gut and skin toxicities while radiosensitising tumour cells and maintaining tumour control compared to RT alone [13-20].

This correlates with retrospective clinical studies in which patients taking statins during RT or chemo-RT for rectal, bladder or prostate cancer had significantly higher rates of pathological complete response (CR), local control and progression-free survival, respectively [21-25]. The findings in three published retrospective studies using pCRT in rectal cancer patients were:

- in 407 patients at the Cleveland Clinic, favourable pathological regression was seen in $65.7 \%$ of statin users vs. $48.7 \%$ of others, $p=0.004$ (multivariate OR 2.25; 95\% CI 1.33-3.82) [22].

- multivariate analysis of 891 Canadian patients from multiple centres showed a significantly higher pathological CR rate in statin users (OR 1.7, 95\% CI 1.04-2.89, $p=0.044)$ [23];

- in 349 patients from Memorial Sloan-Kettering Cancer Center pathological CR was higher in statin users ( $30 \%$ vs. $17 \%)$, with multivariate OR 4.2 (95\% CI $1.7-12.1 ; p=0.003$ ) [24].

Furthermore, in a prospective observational study of 308 patients treated with radical pelvic RT at the Royal 
Marsden Hospital, London, those taking statins had significantly reduced RT-induced bowel toxicity, both during treatment $(p=0.04)$ and 1 year later [26].

In contrast two smaller studies have not shown an apparent benefit. A retrospective New Zealand study of 129 rectal cancer patients showed neither reduced acute toxicity nor improved pathological CR rates in the $23 \%$ of patients who took statins during pCRT [27]. A US singlearm phase 2 trial recruited 53 prostate cancer patients to take lovastatin $20-80 \mathrm{mg}$ daily for 1 year starting during external beam RT and/or brachytherapy to prevent late RT-induced rectal injury [28]. Persistent gastrointestinal symptoms at 2 years were seen in $32 \%$ of patients (grade 2 in 6\%), which did not meet the primary endpoint. Furthermore no benefit from statins was seen in a meta-analysis of surgery for prostate cancer $[25,29]$, nor in a trial of adjuvant chemotherapy for colon cancer [30].

\section{Summary of the known and potential risks and benefits to human participants}

The statin chosen for this trial, simvastatin (SIM), is a well-known and widely available HMG-CoA reductase inhibitor commonly used in the treatment of hypercholesterolaemia and ischaemic heart disease. In retrospective studies it reduces recurrence in breast cancer patients [31] and preclinical studies have demonstrated its beneficial interactions with RT. [32, 33] It is very well-tolerated in the majority of patients with $<2 \%$ of patients in clinical trials discontinuing simvastatin due to adverse events. The most common side effects include abdominal pain, diarrhoea, indigestion, and weakness. Rarer side effects include joint pain, memory loss, myalgia, and muscle cramps. In patients taking statins longterm there have been reports of hepatitis, rhabdomyolysis and myositis however these complications are rare $(<$ $1 \%$ of patients). Serious allergic reactions to simvastatin are also rare. $40 \mathrm{mg}$ daily is the highest dose welltolerated [34].

\section{Rationale for trial endpoints mrTRG and pathTRG}

MRI-based tumour regression grading (mrTRG), a 5point system validated by the MERCURY group [35], is used for the primary endpoint in this trial as it permits much more sensitive and reliable preoperative assessment of tumour regression following pCRT in rectal cancer patients than other methods used currently (endoscopy, CT, PET-CT, endoluminal ultrasound or routine MRI) $[36,37]$. mrTRG has proven to be a good predictor of pathological tumour regression grading (pathTRG) after pCRT [38], as well as independently predicting DFS and OS [39]. In a separate study, mrTRG identified ten times as many pathological CR patients as clinical inspection of the tumour following pCRT, with no compromise of the false-positive rate [37].

The rate of favourable (grades 1-2) mrTRG is the primary endpoint for the SPAR trial, based on three recently-published pCRT rectal cancer trials [40, 41]. In the MERCURY-II trial [40] favourable (grades 1-2), intermediate (grade 3) and unfavourable mrTRG (grades 4-5) had 3-year DFS of 82, 72 and 61\% respectively (G Brown, personal communication). The phase 2 EXPERT and EXPERT-C trials evaluated neoadjuvant chemotherapy followed by pCRT [41]. On pooled analysis of these trials, mrTRG performed 4 weeks after completion of pCRT was evaluable in $85.5 \%$ of 269 patients; favourable (mrTRG 1-2), intermediate (mrTRG 3) and unfavourable (mrTRG 4-5) outcomes were seen in 41.7, 30.9 and $27.4 \%$, respectively. pathTRG was evaluable in $86.9 \%$ of 244 resected patients with favourable, intermediate and unfavourable scores seen in 35.4, 29.7 and 34.9\% respectively. Favourable mrTRG was independently associated with PFS (HR 0.37, $p<0.001$ ) and OS (HR $0.44, p=0.006$ ) [41].

While the mrTRG training provided in SPAR has been shown to achieve moderate-to-excellent agreement between expert and training radiologists [42], it is important to show independently in the SPAR trial that mrTRG can be successfully and reproducibly performed in multiple centres, and shows a strong correlation with pathTRG and clinically-important cancer outcomes.

While pathological CR with pCRT is associated with the best clinical outcomes, and is commonly considered the "gold standard", the spectrum of response on standardised pathological tumour regression grading (pathTRG) systems correlates with DFS and overall survival (OS), and informs prognosis in the full spectrum of patients, not just the small minority with pathological CR. Thus a 4-tier system has been widely adopted, including in Australasia [43], but a 3-tier system derived from this (grouping the two most favourable grades) shows greater reproducibility (interobserver agreement $\mathrm{K}=0.84)$ and is recommended [44].

SPAR will provide independent validation of the correlation of mrTRG with pathTRG and cancer outcomes, as well as evaluating the reproducibility of mrTRG assessment by $\mathrm{NZ}$ and Australian radiologists (not yet commonly used) and pathTRG by pathologists (standard practice).

Early surrogates for tumour response to pCRT are being investigated in clinical trials, particularly for their potential to modify the extent of surgery, or possibly avoidance of surgery in those who achieve a radiological CR [36]. The importance of this is three-fold: firstly, mrTRG can assist surgeons in planning the extent of surgery based on the response to pCRT; for example, this could change whether a patient needs a permanent stoma or reduce the risk of positive circumferential 
resection margins. Secondly, some patients who appear to have clinical CR after pCRT are electing to avoid surgery, based on much less precise methods of assessing residual disease than mrTRG, so using mrTRG can improve the precision of assessment of likely pathological CR [36, 37]. Thirdly, the poor outcomes of patients with an unfavourable response to pCRT has led to proposals to use mrTRG to identify this group in clinical trials and evaluate if adding other treatment (such as different chemotherapy or biological therapy) prior to surgery can improve cancer outcomes (e.g. The TRIGGER Study; clinicaltrials.gov No. NCT02704520).

\section{Timing of post-pCRT MRI}

In the SPAR study, the second MRI is scheduled at 6-8 weeks after pCRT to allow for surgery at 7-12 weeks. Surgery is now commonly delayed to $10-12$ weeks after completion of pCRT in anticipation of improved tumour regression with additional time after pCRT [45]. There are conflicting reports as to whether this is the case, based on pathological CR rates [46-48]. However, while the pathological changes in the tumour following pCRT are expected to evolve over many weeks, this may not improve DFS or PFS as these outcomes are most likely determined by the inherent tumour sensitivity to pCRT. This is supported by a retrospective Korean study of 1786 patients treated with pCRT for locally-advanced rectal cancer, in whom pathological CR rates were highest when surgery was performed 5-10 weeks after pCRT; those who had resection delayed to $>7$ weeks after pCRT had significantly higher pathological CR rates but no difference in relapse-free or overall survival [49]. Of concern, a French prospective randomised controlled trial of surgery at 7 or 11 weeks after pCRT in 265 rectal cancer patients showed no significant difference in the primary endpoint of pathological CR rates but there was significantly higher post-operative morbidity and poorer quality of mesorectal excision in the 11 week group, possibly due to greater RT-induced fibrosis [47]. A retrospective US study in 6397 patients evaluating time between RT and resection for rectal cancer found that an interval $>60$ days was significantly associated with inferior survival, lower rates of sphincter-preserving surgery and an increased rate of positive surgical margins [48]. This suggests that surgery could be optimally performed earlier than commonly practised currently, and SPAR will accommodate this range.

\section{Translational endpoints}

This trial is an important opportunity to identify whether assessment of systemic inflammation (reflected in the modified Glasgow prognostic score, mGPS) and the local inflammatory response (through characterising infiltrating lymphocytes) could be an important translational research component of a subsequent phase III trial of statins in rectal cancer patients. Additionally, it will allow us to evaluate the impact of SIM on the relationship between specific Tcell infiltrates in pre-pCRT biopsies and pathTRG in the resected tumours, and with normal tissue inflammation post-pCRT.

It is long-recognised that a local inflammatory response with infiltration of T-lymphocytes into CRC carries a better prognosis, independent of tumour stage [50, 51]. Conversely preoperative systemic inflammation, reflected in the blood neutrophil/lymphocyte ratio (NLR) or serum creactive protein (CRP) and albumin levels (summarised in the modified Glasgow Prognostic Score), correlates with worse prognosis, independent of stage and preoperative therapy [52]. This adverse prognostic relationship persists with elevated mGPS 3-6 months postoperatively [52]. While statins are anti-inflammatory [12] and reduce the NLR in patients with high cholesterol [53], it is not known whether they lower the NLR or mGPS in cancer patients.

The Immunoscore is a recently-validated test that characterises $\mathrm{T}$-cell subsets infiltrating into the centre of the tumour and at the invasive margin of CRC by immunohistochemistry (IHC) for CD3 and CD8; higher scores correlate with a lower risk of relapse and improved DFS and OS, independently of stage $[54,55]$. While the Immunoscore was validated in rectal cancer patients who had primary surgery, it cannot be applied to those patients who received pCRT because identification of central and margin regions is compromised due to tumour regression and fibrosis [56]. However, in diagnostic rectal biopsies taken prior to pCRT prominent infiltration of CD3+ and CD8+ T-cells strongly correlated with pathological CR rates following pCRT (56).

Statins have complex effects on T-cell biology, including induction of regulatory $\mathrm{T}$-cells (Tregs), their migration into tumours and inhibition of the induction of Th1 and Th17 cells [57]. Tregs, which dampen immune responses, can differentiate into effector Tregs (eTregs); these display markers of both immune suppression and activation [58] and are associated with a positive patient outcome in CRC [59]. However, Treg populations in lymph nodes do not correlate with patient outcome, unlike their presence in the primary colorectal tumour [60].

\section{Overall purpose}

The overall purpose of this trial is to determine the effect of SIM on outcomes of pCRT for rectal cancer. Outcomes will be evaluated both by tumour regression as well as the tolerability of pCRT, estimating the size of the benefit by MRI-based assessment as well as pathological assessment of tumour regression. In addition, we will examine the biological mechanisms involved. The study will also assess the reproducibility of assessing mrTRG and pathTRG by radiologists and pathologists in 
Australia and New Zealand. Furthermore, the information provided by the post-CRT mrTRG could influence the intended surgical plan and optimise timing of surgery depending on tumour response to pCRT.

A positive outcome, either improved tumour regression or reduced toxicity from pCRT or both, would lead to a larger phase III trial to confirm these findings. It may also lead to evaluation of statins in prospective trials in many other settings where radiation is used in cancer treatment. This trial is an important opportunity to identify whether the addition of SIM to pCRT significantly modulates systemic inflammation (reflected in the NLR and mGPS) and the local inflammatory response (through characterising infiltrating immune cells). If so, it would inform the inclusion of these translational research components in a subsequent phase III trial of statins in rectal cancer patients. It will also allow us to evaluate the impact of SIM on the correlation of T-cell infiltrates in pre-pCRT biopsies with pathTRG in the resected tumours, and with normal tissue inflammation after pCRT.

\section{Methods/design}

SPAR is a randomised phase 2 study with the overall aim to evaluate the effect of SIM on efficacy and toxicity of PCRT in rectal cancer patients, and on systemic and local inflammatory responses. Recruitment of 222 patients is required to address the primary objective. The study will recruit patients from participating AGITG hospitals/institutions in Australia and New Zealand.

Primary Objective:

The primary objective is to compare rates of favourable (grades 1-2) mrTRG (by central review) following PCRT with SIM versus with placebo, considering mrTRG in 4 ordered categories: $1,2,3,4-5$ (proportion of patients with favourable mrTRG in SIM and placebo groups). mrTRG will be assessed by comparison of the MR scan taken after pCRT with the scan before pCRT, which will be performed using the MERCURY protocol (38), and images analysed as described [38].

Secondary Objectives:

Secondary objectives are to compare between the SIM and placebo groups treated with pCRT: 1) The rate of favourable (grades 1-2) pathTRG in resected tumours by central review (proportion of patients with favourable pathTRG at surgical resection); 2) The incidence of $\geq$ grade 2 acute GI and non-GI adverse events, assessed using CTCAE version 4.03 ; 3) The incidence of late GI adverse events; 4) Compliance with intended pCRT (proportion of patients completing $\geq 90 \%$ of planned pCRT without dose reductions or delays); 5) Compliance with trial medication (proportion of patients receiving $\geq$ $90 \%$ of the planned trial medication); 6) The proportion of patients undergoing surgical resection post-pCRT; 7) 3-year local recurrence (LR) rate, disease-free survival (DFS) and cancer-specific survival (CSS) (3-year LR rate, DFS and CSS); 8) the pathological scores determined by the central pathologist for radiation colitis in irradiated rectum in the resected specimen (radiation colitis scores).

Translational Science Objectives:

A number of correlative/translational objectives are intended. We aim to determine in the total trial population: 1) The association between mrTRG and pathTRG grouping (association between mrTRG and pathTRG grouping); 2) The inter-observer agreement between site radiologists and a central radiologist on mrTRG scoring (inter-observer agreement on mrTRG scoring); 3) The inter-observer agreement between site pathologists and a central pathologist on pathTRG scoring (inter-observer agreement on pathTRG scoring). In addition we aim to compare between the SIM and placebo groups treated with pCRT: 1) The association between CD3+ and/or CD8+ T-cell infiltrates in the tumour in the pre-pCRT diagnostic biopsies and pathTRG (association between $\mathrm{T}$ cell infiltrates and pathTRG);2) the intensity and distribution of subsets of infiltrating T-cells in irradiated normal and malignant tissue in the resected specimen; 3) The influence of SIM on systemic inflammation, assessed with the mGPS and the NLR (mGPS and NLR).

\section{Design}

SPAR is a randomised, double-blind, placebo-controlled, multicentre phase II trial. Eligible patients will be allocated to one of two treatment groups (SIM or placebo) in a 1:1 ratio (Fig. 1: SPIRIT diagram).

Treatment allocation will be balanced using minimisation for the following characteristics:

- trial site

- AJCC clinical T stage (<4 vs. 4$)$

- AJCC clinical $N$ stage (<2 vs. 2$)$

- the presence of either mesorectal fascia involvement (tumour margin within $1 \mathrm{~mm}$ of the fascia) or lymphovascular space invasion (including extramural venous invasion) on MRI

\section{Eligibility criteria}

The target population is adult patients with biopsyproven rectal adenocarcinoma (or high-grade dysplasia on biopsy with radiological evidence of invasive tumour) planned for concurrent long-course pCRT using a standard fluoropyrimidine-based chemotherapy schedule.

\section{Inclusion criteria}

1. Males or females with biopsy proven rectal adenocarcinoma, or high-grade dysplasia with radiological evidence of invasive tumour. 


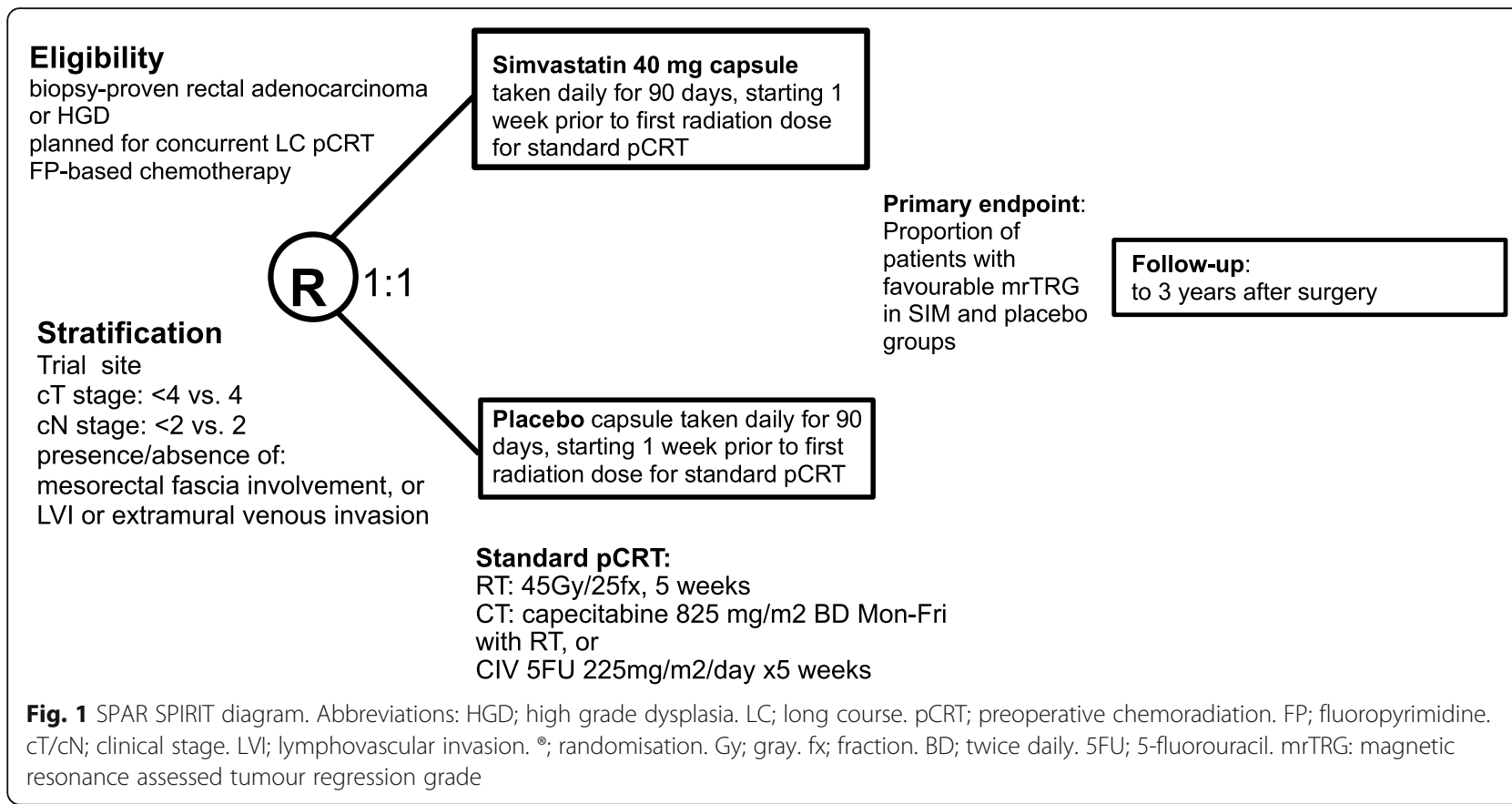

2. Distal border of the tumour is below the peritoneal reflection as assessed by MRI scan.

3. Age $\geq 18$ years.

4. Clinical TNM tumour staging is T2-4 N0-2 M0 after staging investigations including CT scan of chest, abdomen and pelvis and pelvic MRI scan. Patients with resectable M1 (e.g. oligometastatic liver or lung) disease who are being treated with curative intent can be eligible, following approval from the SPAR Study Chair.

5. Planned for concurrent long-course pCRT using fluoropyrimidine-based chemotherapy

6. Radiologically measurable disease on baseline pelvic MRI scan.

7. Adequate bone marrow, liver and renal function (platelets $>100 \times 10^{9} / \mathrm{L}$, neutrophils $>1.5 \times 10^{9} / \mathrm{L}$, ALT/AST $<3 \times$ ULN, bilirubin $<1.5 \times$ ULN, estimated creatinine clearance $>50 \mathrm{ml} / \mathrm{min}$ ).

8. Trial treatment planned to start within 28 days of randomisation.

9. Diagnostic biopsy of rectal tumour is available for histological substudies.

10. Willing and able to comply with all trial requirements.

11. Signed, written informed consent for the main trial.

\section{Exclusion criteria}

1. Contraindications or hypersensitivity to statins, fluoropyrimidine chemotherapy or radiotherapy
2. Patients planned to receive oxaliplatin or biological agents (e.g. cetuximab) as part of pCRT

3. Taking statins in the 6 weeks before planned start of pCRT

4. Predicted life expectancy of less than 3 years

5. Prior pelvic or rectal radiotherapy

6. History of another malignancy within 5 years prior to registration (not including adequately treated carcinoma-in-situ, basal cell carcinoma of the skin, squamous cell carcinoma of the skin, or superficial transitional cell carcinoma of the bladder). Patients with a history of other malignancies are eligible if they have been continuously disease free for at least 5 years after definitive primary treatment

7. Concurrent illness, including severe infection that may jeopardise the ability of the patient to undergo the procedures outlined in this protocol with reasonable safety

8. Serious medical or psychiatric conditions that might limit the ability of the patient to comply with the protocol

9. Pregnancy, lactation, or inadequate contraception.

\section{Treatment}

Simvastatin or placebo is the trial intervention. Concurrent preoperative chemoradiation (pCRT) using fluoropyrimidine-based chemotherapy and radiotherapy is required standard concomitant treatment (Fig. 1).

One simvastatin $40 \mathrm{mg}$ capsule or one matching placebo capsule taken orally each evening, will commence 7 (+/3) days prior to pCRT and continued for 90 consecutive 
days. Subject compliance with the trial treatment will be determined at protocol-specified assessments by questioning the participant and a formal count of the capsules returned at completion of study treatment (6 weeks after pCRT).

Trial treatment (SIM or placebo) will be permanently discontinued if progressive disease (PD) is documented, unacceptable treatment-related toxicity occurs, a delay of pCRT of $>28$ days occurs, the clinician believes that continuation of trial treatment is not in the patient's best interest, or the patients fails to comply or declines further treatment.

\section{Radiation therapy}

It is recommended to follow eviQ Rectal (Neoadjuvant EBRT Chemoradiation Pre-operative Long Course) Guidelines version 2 or later (www.eviq.org.au).

Clinical Target Volume A (CTV A) is defined as per the recent international consensus guidelines [61]. The Planning Target Volume (PTV) A margin should be 0.7 to $1.0 \mathrm{~cm}$, except at skin, where planning system requirements mandate it be trimmed to $2-5 \mathrm{~mm}$ within the skin surface. The dose to PTV A is $45 \mathrm{~Gy}$ in 25 fractions, 1.8Gy per day, 5 days per week.

Clinical Target Volume B (CTV B) includes the mesorectum and pre-sacral region at involved levels with a 1-2 $\mathrm{cm}$ margin cephalad and 1 to $2 \mathrm{~cm}$ on gross tumour within the rectum. The PTV B margin should be 0.7 to $1.0 \mathrm{~cm}$, except at skin, where planning system requirements mandate it be trimmed to $2-5 \mathrm{~mm}$ within the skin surface.

For 3-dimensional conformal radiation therapy (3DCRT), a tumour boost of $5.4 \mathrm{~Gy}$ at $1.8 \mathrm{~Gy}$ per fraction to PTV B (50.4Gy cumulative including contribution from PTV A) is required for patients with $\mathrm{T} 3$ tumours. A boost dose up to $10.8 \mathrm{~Gy}$ at $1.8 \mathrm{~Gy}$ per fraction (55.8Gy cumulative, including contribution from PTV A) is allowed for patients with T4 fixed cancer and high-risk T3 tumours.

For intensity modulated radiation therapy (IMRT) or volumetric modulated arc therapy (VMAT), simultaneous boost technique is recommended. Total cumulative dose to PTV B is to be 50 Gy.

Permission for other radiation techniques aiming to deliver equivalent radiation dose should be obtained in advance from Trial Management Committee.

\section{Chemotherapy}

The accepted chemotherapy and dosing regimens [6] are one of:

- capecitabine $825 \mathrm{mg} / \mathrm{m}^{2} /$ day PO BID 5 days a week on days of RT administration

- capecitabine $825 \mathrm{mg} / \mathrm{m}^{2} /$ day PO BID 7 days a week for the duration of RT
- 5-fluorouracil $225 \mathrm{mg} / \mathrm{m}^{2} /$ day via continuous venous infusion for the duration of RT

- Other therapies and/or dosing regimen that have been accepted as standard of care in Australia and New Zealand may be allowed following agreement from the Study Chair

A maximum BSA of $2.2 \mathrm{~m}^{2}$ is recommended for dosing of fluoropyrimidine-based chemotherapy. Clinicians must pre-specify which schedule they will be using for each participant. Once allocated, the patients must then adhere to the specified schedule throughout the treatment period, unless modified for safety reasons.

\section{Dose modifications and supportive therapies}

Patients should be managed with pCRT according to institutional protocols, including dose modifications and delays for treatment-related toxicities. Recommendations are provided for guidance on management of toxicities related to pCRT and are defined in the protocol. If pCRT is discontinued, trial treatment should continue unless unacceptable trial treatment toxicity is observed. Other concomitant medications and supportive therapies are permitted, and there is a list of prohibited medications (drugs that may interact with the trial drug) in the detailed protocol.

\section{Surgery}

Patients will undergo resection of their rectal cancer at a time recommended by their surgeon (generally 7-10 weeks after completion of pCRT). Patients with an excellent clinical and radiological response may be observed under a "watchful waiting" programme if agreed with their surgeon.

Surgery may include open, laparoscopic, robotic or transanal total mesorectal excision approach. This will include both restorative low anterior resection, or abdominoperineal resection with permanent end-colostomy.

\section{Post-operative management}

Treatment after discontinuation of trial treatment is at the discretion of the patient's clinician. Adjuvant chemotherapy may be administered on the recommendation of the treating clinician and its use will be recorded.

Blood tests will be performed on the first day of pCRT, prior to starting chemotherapy, to assess the impact of trial medication on the mGPS and NLR. Clinical assessments including IBDQ-B questionnaire will be conducted on Trial Weeks 3, 5 and 7 [62].

Week 13 assessments will be performed 6 weeks (+/7 days) after completion of pCRT and prior to surgery. An MRI scan for mrTRG must be completed 6-8 weeks after completion of pCRT and must be prior to surgery. The postoperative visit will include assessment of length 
of hospital stay after surgery and any readmission data. Subsequent follow-up is annually.

\section{Data to be collected}

The study will collect data on: baseline patient and tumour status; treatment delivery; baseline and postpCRT MRI assessments; clinical and laboratory toxicity assessments; baseline and resection histopathology; followup data for PFS and OS (Table 1).

\section{Statistical methodology Sample size}

The primary endpoint of this trial is rates of favourable mrTRG (grades 1 or 2). This will be analysed using ordinal regression with 4 ordered categories of mrTRG (1, 2,3 and 4-5) to provide greater sensitivity. Based on published data using MRI 6-8 weeks after pCRT, the expected rate of mrTRG in the control group is $9 \%$ grade 1 , 39\% grade 2 , $20 \%$ grade 3 and $32 \%$ grades $4-5$. A $35 \%$ relative increase in favourable mrTRG would be a worthwhile difference to inform a phase III trial.

Based on an ordinal proportional odds assumption, a sample size of 222 patients (111 treated with SIM and 111 controls) will have $>80 \%$ power to detect a change in $\operatorname{mrTRG}$ rates to $17 \%$ grade $1,48 \%$ grade $2,16 \%$ grade 3 and $19 \%$ grades $4-5$ with $95 \%$ confidence.

\section{Statistical analysis}

Intention-to-treat analysis of results will be the primary analysis. In addition, an exploratory per protocol analysis will also be conducted which will exclude ineligible patients, those not taking trial medication when starting pCRT and those who withdraw their consent to participate prior to response evaluation.

The primary endpoint and all secondary endpoints expressed as proportions will be estimated, together with corresponding 95\% confidence intervals based on exact binomial distributions. Kaplan-Meier curves will be calculated for all time-to-event endpoints. Rates at specific points in time (e.g. 3-year local recurrence rate) will be estimated from these Kaplan-Meier curves.

Inter-observer agreement is defined as the degree of agreement in mrTRG (or pathTRG) results when reviewed by the central radiologist (or pathologist) and the reporting site radiologists (or pathologists). Results for each of mrTRG and pathTRG will be reported separately in a $3 \mathrm{X} 3$ grid comparing favourable, intermediate, and unfavourable scores between central and site radiologists and pathologists respectively. Inter-observer agreement will be assessed using a weighted kappa statistic $\geq 0.40$ is defined as moderate agreement. This will be assessed for mrTRG after recruitment of 35 randomised patients to ensure procedural consistency and again at the conclusion of the trial.

Adverse events in each arm will be tabulated and graded according the NCI CTCAE version 4.03.

For analysis of translational objectives, association between $\mathrm{CD} 3+$ and $\mathrm{CD} 8+\mathrm{T}$-cell infiltrates in diagnostic rectal cancer biopsies and pathTRG following pCRT will be evaluated using the chi-square test for trend. The effect of SIM on subsets of T-cells in tumours and normal tissue will be evaluated by comparison of distribution of

Table 1 Schedule of Assessments

\begin{tabular}{|c|c|c|c|c|c|c|c|c|c|c|c|}
\hline \multicolumn{12}{|c|}{ Treatment Period - Trial Week } \\
\hline & Baseline / screening & 1 & 2 & 3 & 4 & 5 & 6 & 7 & 13 & 4-6 weeks post-op & Follow up \\
\hline Informed consent & $x$ & & & & & & & & & & \\
\hline History & $x$ & & & & & & & & & $x$ & \\
\hline Physical exam b & $x$ & & & $x$ & & $x$ & & $x$ & & $x$ & \\
\hline Trial medication start & & $x$ & & & & & & & & & \\
\hline Preoperative CRT & & & $X$ & $x$ & $x$ & $X$ & $x$ & $x$ & & & \\
\hline Concomitant meds & $x$ & & & $x$ & & $x$ & & $x$ & $x$ & & $x$ \\
\hline Trial med compliance & & & & $x$ & & $X$ & & $x$ & $x$ & & \\
\hline Adverse events & & & & $x$ & & $x$ & & $X$ & $x$ & & $x$ \\
\hline Haematology & $x$ & & $x$ & $x$ & $x$ & $x$ & $x$ & $x$ & $x$ & & \\
\hline Chemistry ind. CRP & $x$ & & $X$ & $x$ & $x$ & $x$ & $x$ & $X$ & $x$ & & \\
\hline CEA & $x$ & & & & & & & & & & $x$ \\
\hline Serum pregnancy test & $x$ & & & & & & & & & & \\
\hline Resected rectal tissue & & & & & & & & & & $x$ & \\
\hline CT scan & $x$ & & & & & & & & & & $x$ \\
\hline Pelvic MRI & $x$ & & & & & & & & $x^{\circ}$ & & \\
\hline Lower Gl endoscopy & $x$ & & & & & & & & & & $x$ \\
\hline
\end{tabular}


scores for each cell type in SIM and placebo groups using the chi-square test for trend. Changes in the NLR and mGPS over time will be analysed using repeated measures ANOVA.

\section{Interim analyses and early stopping}

No formal interim analysis for efficacy is planned but review of safety data by the AGITG Independent Safety and Data Monitoring Committee (ISDMC) is planned. No early stopping for larger-than-expected differences in mrTRG rates is planned because this endpoint is a putative surrogate for improved patient outcome. Demonstrating a significant difference in the clinicallyimportant DFS and OS endpoints requires a much larger phase III trial.

\section{Trial governance and confidentiality}

The study is conducted by the Australasian Gastrointestinal Trials Group, in conjunction with the NHMRC Clinical Trials Centre. Formal study oversight is by a Trial Management Committee and an Independent Data and Safety Monitoring Committee.

The study will be conducted according to the Note for Guidance on Good Clinical Practice (CPMP/ICH/ 135/95) annotated with TGA comments (Therapeutic Goods Administration DSEB July 2000) and in compliance with applicable laws and regulations. The study will be performed in accordance with the NHMRC Statement on Ethical Conduct in Human Research 2007, the NHMRC Australian Code for the Responsible Conduct of Research 2007, and the principles laid down by the World Medical Assembly in the Declaration of Helsinki 2008.

The study will be conducted in accordance with applicable Privacy Acts and Regulations. All data generated in this study will remain confidential. All information will be stored securely at the NHMRC Clinical Trials Centre, University of Sydney, and will only be available to people directly involved with the study and who have signed a Confidentiality Agreement.

\section{Discussion}

Little progress has been made in the management of $\mathrm{T} 3$ rectal cancer in the last 10 years. The findings in several retrospective studies that statin use in patients undergoing $\mathrm{PCRT}$ appears to confer higher pathological regression rates, more pathological CR [22-24] and also lower RT toxicity $[16,22-24]$, led us to mount this phase II RCT. We chose to start statin therapy 1 week prior to pCRT to observe for clinical and biochemical effects independent of pCRT, and continue it for 3 months to allow maximum interaction with CRT. The use of a standard dose of simvastatin is pragmatic; 40 mg per day is the highest dose that is well tolerated.
The evaluations are comprehensive, including comparative treatment-induced changes in MR scans, pathological evaluation of the resected specimen, and blood biochemical changes, as well as longer-term tumour outcomes (PFS and OS). With this sample size of 222 patients (111 simvastatin and 111 placebo), we should be able to observe meaningful differences in these parameters if simvastatin has any beneficial effect in the treatment of this disease.

If this study has a positive result, a larger RCT will be needed to confirm and validate the merit of statins in the preoperative management of rectal cancer, especially long-term clinical outcomes. Such a finding could also lead to studies of statins in conjunction with radiation and chemotherapy in a range of other malignancies, as well as further exploration of possible mechanisms of action and interaction of statins with both radiation and chemotherapy. The translational substudies undertaken with this trial will provisionally explore some of these possible mechanisms, and the tissue and data can be made available for further investigations.

This trial is an important opportunity to identify whether assessment of systemic inflammation (reflected in the neutrophil-lymphocyte ratio and the mGPS) and the local inflammatory response (through characterising infiltrating immune cells) could be important translational research components of a subsequent phase III trial of statins in rectal cancer patients. Additionally, it will allow us to evaluate the impact of SIM on the interaction of specific T-cell infiltrates in pre-pCRT biopsies with pathTRG in the resected tumours, and with normal tissue inflammation after pCRT.

Recent reports on the merits of mrTRG to assess effects of pCRT suggest that it is a valid endpoint to use in clinical trials [37-41]. This study will further validate mrTRG as an interim endpoint for assessing treatment benefits in rectal cancer, as well as allow exploration of newer MRI features that might refine the TRG assessment. All MR scans will be collected in digital format and can be made available to other groups for further research.

Surgery is now commonly delayed to $10-12$ weeks after completion of pCRT in anticipation of improved tumour regression with additional time after pCRT. There are conflicting reports as to whether this is the case, based on pathological CR rates [46-48]. However, while the pathological changes in the tumour following pCRT are expected to evolve over many weeks, this may not improve DFS or PFS as these outcomes are most likely determined by the inherent tumour sensitivity to pCRT. This is supported by a retrospective Korean study of 1786 patients treated with pCRT for locally-advanced rectal cancer, in whom pathological $\mathrm{CR}$ rates were 
highest when surgery was performed 5-10 weeks after pCRT; those who had resection delayed to $>7$ weeks later had significantly higher pathological CR rates but no difference in relapse-free or overall survival [49]. Of concern, a French prospective randomised controlled trial of surgery at 7 or 11 weeks after pCRT in 265 rectal cancer patients showed no significant difference in the primary endpoint of pathological CR but there was significantly higher post-operative morbidity and poorer quality of mesorectal excision in the 11-week group, possibly due to greater RT-induced fibrosis [47]. A retrospective US study $(n=6397)$ evaluating time between RT and resection for rectal cancer found that an interval $>60$ days was significantly associated with inferior survival and rates of sphincter-preserving surgery and an increased rate of positive surgical margins [48]. This suggests that surgery could be optimally performed earlier than commonly practised currently. In this SPAR study, surgery is intended at 7-10 weeks after completion of pCRT. The data we collect may contribute to refinement of guidelines about surgery timing after pCRT.

\section{Abbreviations}

5FU: 5-fluorouracil; AJCC: American Joint Committee on Cancer; CR: Complete remission/response; CRC: Colorectal cancer; CRP: C-reactive protein; CSS: Cancer-specific survival; CTV: Clinical target volume;

DFS: Disease-free survival; EBRT: External beam RT; GI: Gastrointestinal; HMGCoA: Hydroxymethylguanosyl coenzyme A reductase; IBDQ-B: Inflammatory bowel disease questionnaire B; IHC: Immunohistochemistry; LR: Local recurrence; mGPS: modified Glasgow prognostic score; MRI: Magnetic resonance imaging; mrTRG: magnetic resonance tumour regression grade; NCI CTCAE: National Cancer Institute Common Terminology Criteria for Adverse Events; NHMRC: National Health and Medical Research Council; NLR: Neutrophil-lymphocyte ratio; OS: Overall survival; pathTRG: pathological tumour regression grade; pCRT: preoperative chemoradiation therapy; PETCT: Positron-emission tomography- computed tomography; PFS: Progressionfree survival; PR: Partial response; PTV: Planning target volume; RT: Radiation therapy; SCRT: Short course radiation therapy; SIM: Simvastatin; Tregs: Regulatory T-cells; US: United States of America

\section{Acknowledgements}

The authors thank all current and future participating site staff for identification, recruitment and management of recruited patients, and all recruited patients for their voluntary contribution to this research. Also: Margot Gorzemann and Kate Wilson (preparation of grant applications); Val Gebski (statistical design); Danielle Miller and Amy Zhong (management of data and overall trial procedures).

\section{Authors' contributions}

Concept: MBJ, SPA. Design: MBJ, SPA, KG, GMG, TJP, JA, JC, FF, SN, AS. Protocol development: MBJ, SPA, KG, DE, WH, GMJ, TJP, CSK, MA, JA, JC, FF, SN, AS, MO. Statistical design and analysis: DE. Data collection and study overall management: MBJ, SPA, KG, GA, GMJ, TJP, CSK, MA, JC, FF, SN, AS, MO. Drafting manuscript: all authors. All authors read and approved the final manuscript.

\section{Funding}

Cancer Council NSW (APP1125958, RG 17-07).

Cancer Australia (APP1125958).

New Zealand Health Research Council (18/233).

Funding bodies had no role in the design of this study and will not have any role during its.

execution, analyses, interpretation of the data, or decision to submit results. All 3 funding bodies utilised independent peer-review processes to evaluate the submission.

\section{Availability of data and materials}

The study is currently accruing. Data and materials will be made available for further research upon request after the primary analysis has been presented and published.

\section{Ethics approval and consent to participate}

Royal Prince Alfred Hospital Health Research Ethics Committee. Protocol no. X-17-0108 and HREC/17/RPAH/158 (date: 4 July 2017).

Signed, written informed consent will be obtained from all patients.

\section{Consent for publication}

(of identifying images or other personal or clinical details of participants that compromise anonymity): not applicable.

\section{Competing interests}

The authors declare that they have no competing interests.

\section{Author details}

${ }^{1}$ Waikato Hospital and Waikato Clinical Campus, University of Auckland, Hamilton, New Zealand. ${ }^{2}$ Dr Jones \& Partners, Eastwood, SA, Australia. ${ }^{3}$ NHMRC Clinical Trials Centre, University of Sydney, Sydney, Australia. ${ }^{4}$ Bankstown-Lidcombe Hospital, Bankstown, Australia. ${ }^{5}$ Christchurch Hospital, Christchurch, New Zealand. ${ }^{6}$ Queen Elizabeth Hospital, University of Adelaide, Adelaide, Australia. ${ }^{7}$ Flinders Medical Centre, Flinders University, Adelaide, Australia. ${ }^{8}$ Waikato Hospital, Hamilton, New Zealand. ${ }^{9}$ Consumer Advisory Panel, Australasian Gastro-Intestinal Trials Group, Sydney, Australia. ${ }^{10}$ Regional Cancer and Blood Centre, Auckland District Health Board, Auckland, New Zealand. ${ }^{11}$ Canterbury District Health Board, Christchurch, New Zealand.

${ }^{12}$ Peter MacCallum Cancer Centre, Melbourne, Australia. ${ }^{13}$ Royal Brisbane Hospital, Brisbane, Australia. ${ }^{14}$ University of Newcastle, Lake Macquarie Private Hospital and Calvary Mater Newcastle Hospital, Newcastle, Australia.

Received: 21 October 2019 Accepted: 26 November 2019

Published online: 17 December 2019

References

1. Ministry of Health. Cancer: new registrations and deaths 2012. Wellington: Ministry of Health; 2015.

2. Keating J, Pater P, Lolohea S, Wickremesekera K. The epidemiology of colorectal cancer: what can we learn from the New Zealand Cancer registry? N Z Med J. 2003;116(1174):U437.

3. Australian Institute of Health and Welfare (AlHW). Australian Cancer Incidence and Mortality (ACIM) books: Rectal Cancer. Canberra: AlHW; 2015.

4. O'Connell MJ, Colangelo LH, Beart RW, Petrelli NJ, Allegra CJ, Sharif S, et al. Capecitabine and oxaliplatin in the preoperative multimodality treatment of rectal cancer: surgical end points from National Surgical Adjuvant Breast and bowel project trial R-04. J Clin Oncol. 2014;32(18):1927-34.

5. Ngan SY, Burmeister B, Fisher RJ, Solomon M, Goldstein D, Joseph D, et al. Randomized trial of short-course radiotherapy versus long-course chemoradiation comparing rates of local recurrence in patients with $\mathrm{T} 3$ rectal cancer: trans-Tasman radiation oncology group trial 01.04. J Clin Oncol. 2012;30(31):3827-33.

6. Allegra CJ, Yothers G, O'Connell MJ, Beart RW, Wozniak TF, Pitot HC, et al. Neoadjuvant 5-fu or capecitabine plus radiation with or without oxaliplatin in rectal cancer patients: a phase III randomized clinical trial. J Natl Cancer Inst. 2015;107(11):djv248.

7. Lee YC, Hsieh CC, Chuang JP. Prognostic significance of partial tumor regression after preoperative chemoradiotherapy for rectal cancer: a metaanalysis. Dis Colon Rectum. 2013;56(9):1093-101.

8. Sclafani F, Peckitt C, Cunningham D, Tait D, Giralt J, Glimelius B, et al. Shortand long-term quality of life and bowel function in patients with mridefined, high-risk, locally advanced rectal cancer treated with an intensified neoadjuvant strategy in the randomized phase 2 EXPERT-C trial. Int J Radiat Oncol Biol Phys. 2015;93(2):303-12.

9. Nielsen SF, Nordestgaard BG, Bojesen SE. Statin use and reduced cancerrelated mortality. N Engl J Med. 2012;367(19):1792-802.

10. Shao YY, Hsu CH, Yeh KH, Chen HM, Yeh YC, Lai CL, et al. Statin use is associated with improved prognosis of colorectal cancer in Taiwan. Clin Colorectal Cancer. 2015;14(3):177-84. 
11. Cardwell CR, Hicks BM, Hughes C, Murray L. Statin use after colorectal cancer diagnosis and survival: a population-based cohort study. J Clin Oncol. 2014;32(28):3177-83.

12. Bardou M, Barkun A, Martel M. Effect of statin therapy on colorectal cancer. Gut. 2010;59(11):1572-85.

13. Gibot L, Follet J, Metges JP, Auvray P, Simon B, Corcos L, et al. Human caspase 7 is positively controlled by SREBP-1 and SREBP-2. Biochem J. 2009; 420(3):473-83.

14. Sassano A, Platanias LC. Statins in tumor suppression. Cancer Lett. 2008; 260(1-2):11-9

15. Gazzerro P, Proto MC, Gangemi G, Malfitano AM, Ciaglia E, Pisanti S, et al. Pharmacological actions of statins: a critical appraisal in the management of cancer. Pharmacol Rev. 2012;64(1):102-46.

16. Fritz G, Brachetti C, Kaina B. Lovastatin causes sensitization of HeLa cells to ionizing radiation-induced apoptosis by the abrogation of $\mathrm{G} 2$ blockage. Int J Radiat Biol. 2003;79(8):601-10

17. Nubel T, Damrot J, Roos WP, Kaina B, Fritz G. Lovastatin protects human endothelial cells from killing by ionizing radiation without impairing induction and repair of DNA double-strand breaks. Clin Cancer Res. 2006; 12(3 Pt 1):933-9.

18. Holler V, Buard V, Gaugler MH, Guipaud O, Baudelin C, Sache A, et al. Pravastatin limits radiation-induced vascular dysfunction in the skin. J Invest Dermatol. 2009;129(5):1280-91.

19. Haydont V, Gilliot O, Rivera S, Bourgier C, Francois A, Aigueperse J, et al. Successful mitigation of delayed intestinal radiation injury using pravastatin is not associated with acute injury improvement or tumor protection. Int J Radiat Oncol Biol Phys. 2007;68(5):1471-82.

20. Follet J, Corcos L, Baffet G, Ezan F, Morel F, Simon B, et al. The association of statins and taxanes: an efficient combination trigger of cancer cell apoptosis. Br J Cancer. 2012;106(4):685-92.

21. Tsai HK, Katz MS, Coen JJ, Zietman AL, Kaufman DS, Shipley WU. Association of statin use with improved local control in patients treated with selective bladder preservation for muscle-invasive bladder cancer. Urology. 2006; 68(6):1188-92.

22. Mace AG, Gantt GA, Skacel M, Pai R, Hammel JP, Kalady MF. Statin therapy is associated with improved pathologic response to neoadjuvant chemoradiation in rectal cancer. Dis Colon Rectum. 2013;56(11):1217-27.

23. Armstrong D, Raissouni S, Price HJ, Mercer J, Powell E, MacLean A, et al. Predictors of pathologic complete response after neoadjuvant treatment for rectal cancer: a multicenter study. Clin Colorectal Cancer. 2015;14(4):291-5.

24. Katz MS, Minsky BD, Saltz LB, Riedel E, Chessin DB, Guillem JG. Association of statin use with a pathologic complete response to neoadjuvant chemoradiation for rectal cancer. Int J Radiat Oncol Biol Phys. 2005;62(5):1363-70.

25. Kollmeier MA, Katz MS, Mak K, Yamada Y, Feder DJ, Zhang Z, et al. Improved biochemical outcomes with statin use in patients with high-risk localized prostate cancer treated with radiotherapy. Int J Radiat Oncol Biol Phys. 2011;79(3):713-8

26. Wedlake L, Silia F, Benton B, Lalji A, Thomas K, Dearnaley DP, et al. Evaluating the efficacy of statins and ACE-inhibitors in reducing gastrointestinal toxicity in patients receiving radiotherapy for pelvic malignancies. Eur J Cancer. 2012;48(14):2117-24.

27. Hardie $C$, Jung $Y$, Jameson M. Effect of statin and aspirin use on toxicity and pathological complete response rate of neo-adjuvant chemoradiation for rectal cancer. Asia Pac J Clin Oncol. 2016;12(2):167-73.

28. Anscher MS, Chang MG, Moghanaki D, Rosu M, Mikkelsen RB, Holdford D, et al. A phase ii study to prevent radiation-induced rectal injury with lovastatin. Am J Clin Oncol. 2018;41(6):544-8.

29. Scosyrev E, Tobis S, Donsky H, Wu G, Joseph J, Rashid H, et al. Statin use and the risk of biochemical recurrence of prostate cancer after definitive local therapy: a meta-analysis of eight cohort studies. BJU Int. 2013;111(3 Pt B):E71-7.

30. Ng K, Ogino S, Meyerhardt JA, Chan JA, Chan AT, Niedzwiecki D, et al. Relationship between statin use and colon cancer recurrence and survival: results from CALGB 89803. J Natl Cancer Inst. 2011;103(20):1540-51.

31. Ahern TP, Pedersen L, Tarp M, Cronin-Fenton DP, Garne JP, Silliman RA, et al. Statin prescriptions and breast cancer recurrence risk: a Danish nationwide prospective cohort study. J Natl Cancer Inst. 2011;103(19):1461-8.

32. Lacerda L, Reddy JP, Liu D, Larson R, Li L, Masuda H, et al. Simvastatin radiosensitizes differentiated and stem-like breast cancer cell lines and is associated with improved local control in inflammatory breast cancer patients treated with postmastectomy radiation. Stem Cells Transl Med 2014;3(7):849-56.
33. Zhao X, Yang H, Jiang G, Ni M, Deng Y, Cai J, et al. Simvastatin attenuates radiation-induced tissue damage in mice. J Radiat Res. 2014:55(2):257-64.

34. Abd TT, Jacobson TA. Statin-induced myopathy: a review and update. Expert Opin Drug Saf. 2011;10(3):373-87.

35. Patel UB, Blomqvist LK, Taylor F, George C, Guthrie A, Bees N, et al. MRI after treatment of locally advanced rectal cancer: how to report tumor response -- the MERCURY experience. AJR Am J Roentgenol. 2012;199(4):W486-95.

36. Zhao RS, Wang H, Zhou ZY, Zhou Q, Mulholland MW. Restaging of locally advanced rectal cancer with magnetic resonance imaging and endoluminal ultrasound after preoperative chemoradiotherapy: a systemic review and meta-analysis. Dis Colon Rectum. 2014;57(3):388-95.

37. Bhoday J, Smith F, Siddiqui MR, Balyasnikova S, Swift RI, Perez R, et al. Magnetic resonance tumor regression grade and residual mucosal abnormality as predictors for pathological complete response in rectal cancer postneoadjuvant chemoradiotherapy. Dis Colon Rectum. 2016:59(10):925-33.

38. Patel UB, Brown G, Rutten H, West N, Sebag-Montefiore D, Glynne-Jones R, et al. Comparison of magnetic resonance imaging and histopathologica response to chemoradiotherapy in locally advanced rectal cancer. Ann Surg Oncol. 2012:19(9):2842-52.

39. Patel UB, Taylor F, Blomqvist L, George C, Evans H, Tekkis P, et al. Magnetic resonance imaging-detected tumor response for locally advanced rectal cancer predicts survival outcomes: MERCURY experience. J Clin Oncol. 2011; 29(28):3753-60.

40. Battersby NJ, How P, Moran B, Stelzner S, West NP, Branagan G, et al. Prospective validation of a low rectal cancer magnetic resonance imaging staging system and development of a local recurrence risk stratification model: the MERCURY II study. Ann Surg. 2016;263(4):751-60.

41. Sclafani F, Brown G, Cunningham D, Wotherspoon A, Tait D, Peckitt C, et al. PAN-EX: a pooled analysis of two trials of neoadjuvant chemotherapy followed by chemoradiotherapy in MRI-defined, locally advanced rectal cancer. Ann Oncol. 2016;27(8):1557-65.

42. Siddiqui MR, Gormly KL, Bhoday J, Balyansikova S, Battersby NJ, Chand M, et al. Interobserver agreement of radiologists assessing the response of rectal cancers to preoperative chemoradiation using the MRI tumour regression grading (mrTRG). Clin Radiol. 2016;71(9):854-62.

43. Royal College of Pathologists of Australasia. Colorectal Cancer structured reporting protocol (2nd edition). Surry Hills: RCPA; 2016.

44. Ryan R, Gibbons D, Hyland JMP, Treanor D, White A, Mulcahy HE, et al. Pathological response following long-course neoadjuvant chemoradiotherapy for locally advanced rectal cancer. Histopathology. 2005 47(2):141-6.

45. Habr-Gama A, Perez RO, Proscurshim I. Nunes dos Santos RM, kiss D, GamaRodrigues J, et al. interval between surgery and neoadjuvant chemoradiation therapy for distal rectal cancer: does delayed surgery have an impact on outcome? Int J Radiat Oncol Biol Phys. 2008;71(4):1181-8.

46. Evans J, Bhoday J, Sizer B, Tekkis P, Swift R, Perez R, et al. Results of a prospective randomised control 6 vs 12 trial: Is greater tumour downstaging observed on post treatment MRI if surgery is delayed to 12weeks versus 6-weeks after completion of neoadjuvant chemoradiotherapy? Ann Oncol. 2016;27(Suppl 6):vi149.

47. Lefevre $J H$, Mineur L, Kotti S, Rullier E, Rouanet $P$, de Chaisemartin $C$, et al. Effect of interval (7 or 11 weeks) between neoadjuvant radiochemotherapy and surgery on complete pathologic response in rectal cancer: a multicenter, randomized, controlled trial (GRECCAR-6). J Clin Oncol. 2016; 34(31):3773-80

48. Huntington CR, Boselli D, Symanowski J, Hill JS, Crimaldi A, Salo JC. Optimal timing of surgical resection after radiation in locally advanced rectal adenocarcinoma: an analysis of the national cancer database. Ann Surg Oncol. 2016:23(3):877-87.

49. Kwak YK, Kim K, Lee JH, Kim SH, Cho HM, Kim DY, et al. Timely tumor response analysis after preoperative chemoradiotherapy and curative surgery in locally advanced rectal cancer: a multi-institutional study for optimal surgical timing in rectal cancer. Radiother Oncol. 2016;119(3):512-8.

50. Pages F, Berger A, Camus M, Sanchez-Cabo F, Costes A, Molidor R, et al. Effector memory T cells, early metastasis, and survival in colorectal cancer. N Engl J Med. 2005;353(25):2654-66.

51. Roxburgh CS, McMillan DC. The role of the in situ local inflammatory response in predicting recurrence and survival in patients with primary operable colorectal cancer. Cancer Treat Rev. 2012;38(5):451-66.

52. Guthrie GJ, Roxburgh CS, Farhan-Alanie OM, Horgan PG, McMillan DC Comparison of the prognostic value of longitudinal measurements of 
systemic inflammation in patients undergoing curative resection of colorectal cancer. Br J Cancer. 2013;109(1):24-8.

53. Akin F, Ayca B, Kose N, Sahin I, Akin MN, Canbek TD, et al. Effect of atorvastatin on hematologic parameters in patients with hypercholesterolemia. Angiology. 2013;64(8):621-5.

54. Galon J, Mlecnik B, Marliot F, Ou F-S, Bifulco C, Lugli A, et al. Validation of the Immunoscore (IM) as a prognostic marker in stage I/I/III colon cancer: results of a worldwide consortium-based analysis of 1,336 patients. J Clin Oncol. 2016;34(Suppl):abstr 3500.

55. Galon J, Mlecnik B, Bindea G, Angell HK, Berger A, Lagorce C, et al. Towards the introduction of the 'Immunoscore' in the classification of malignant tumours. J Pathol. 2014;232(2):199-209.

56. Anitei MG, Zeitoun G, Mlecnik B, Marliot F, Haicheur N, Todosi AM, et al. Prognostic and predictive values of the immunoscore in patients with rectal cancer. Clin Cancer Res. 2014:20(7):1891-9.

57. Forero-Pena DA, Gutierrez FR. Statins as modulators of regulatory T-cell biology. Mediat Inflamm. 2013;2013:167086.

58. Cretney E, Kallies A, Nutt SL. Differentiation and function of Foxp3(+) effector regulatory T cells. Trends Immunol. 2013;34(2):74-80.

59. Ladoire S, Martin F, Ghiringhelli F. Prognostic role of FOXP3+ regulatory T cells infiltrating human carcinomas: the paradox of colorectal cancer. Cancer Immunol Immunother. 2011;60(7):909-18.

60. Kemp RA, Black MA, McCall J, Yoon HS, Phillips V, Anjomshoaa A, et al. T cell subpopulations in lymph nodes may not be predictive of patient outcome in colorectal cancer. J Exp Clin Cancer Res. 2011;30:78.

61. Valentini V, Gambacorta MA, Barbaro B, Chiloiro G, Coco C, Das P, et al. International consensus guidelines on clinical target volume delineation in rectal cancer. Radiother Oncol. 2016;120(2):195-201.

62. Khalid U, McGough C, Hackett C, Blake P, Harrington KI, Khoo VS, et al. A modified inflammatory bowel disease questionnaire and the Vaizey incontinence questionnaire are more sensitive measures of acute gastrointestinal toxicity during pelvic radiotherapy than RTOG grading. Int J Radiat Oncol Biol Phys. 2006;64(5):1432-41.

\section{Publisher's Note}

Springer Nature remains neutral with regard to jurisdictional claims in published maps and institutional affiliations.

Ready to submit your research? Choose BMC and benefit from:

- fast, convenient online submission

- thorough peer review by experienced researchers in your field

- rapid publication on acceptance

- support for research data, including large and complex data types

- gold Open Access which fosters wider collaboration and increased citations

- maximum visibility for your research: over $100 \mathrm{M}$ website views per year

At $\mathrm{BMC}$, research is always in progress.

Learn more biomedcentral.com/submissions 